\title{
Exigência em fósforo disponível para alevinos de tilápia do Nilo
}

\author{
Dietary phosphorus requirement for fingerlings Nile Tilápia
}

\author{
Luiz Edivaldo Pezzato ${ }^{1}$ Maria Julia Santa Rosa ${ }^{2}$ \\ Margarida Maria Barros $^{3}$ Igo Gomes Guimarães ${ }^{4}$
}

\section{RESUMO}

O fósforo se destaca como um dos minerais mais importantes na nutrição animal. Apresenta-se pouco disponível em alimentos de origem vegetal por estar quimicamente ligado a outros minerais e nutrientes, em que se denomina fósforo fítico ou fitato. Desta forma, objetivou-se avaliar diferentes níveis de fósforo disponível em rações para alevinos de tilápia do Nilo (Oreochromis niloticus), por meio do ganho em peso, do consumo de ração aparente, da conversão alimentar aparente, da eficiência alimentar, da sobrevivência e da composição química da carcaça. O delineamento experimental foi inteiramente casualizado, com seis tratamentos $(0 ; 0,25$; 0,50; 0,75; 1,00 e 1,25\% de fósforo disponível) e cinco repetições. As rações, utilizadas para avaliar os diferentes níveis de fósforo disponível, foram formuladas exclusivamente com alimentos de origem vegetal, sendo isoprotéicas (30,0\% PD), isoenergéticas (3.300 kcal de ED $\mathrm{kg}^{-1}$ ) e contendo a mesma relação cálcio:fósforo. Conclui-se que, para alevinos de tilápia do Nilo, o nível mínimo de 0,75\% de fósforo disponível nas rações proporciona melhor desempenho produtivo $e$ composição química da carcaça.

Palavras-chave: minerais, fitato, peixes, nutrição, Oreochromis niloticu.

\section{ABSTRACT}

Phosphorus is considered one of the most important mineral in animal nutrition. In vegetables feedstuffs this mineral can be less available because his chemistry connection with other minerals and nutrients, named the phosphorus phytic or phytate. Thus this research was aimed evaluating different levels of available phosphorus in diets for Nile tilapia (Oreochromis niloticus) fingerlings based on weight gain, feed consumption, feed conversion ratio, feed efficiency, survival and body composition. The experimental design was completely randomized with six treatment $(0,0.25,0.50,0.75$, 1.00 e $1.25 \%$ of available phosphorus) and five replicates. The rations used to evaluate the different levels of available phosphorus were exclusively formulated, using vegetables feedstuffs, to contain $30.0 \% \mathrm{PD}, 3300 \mathrm{kcal}$ of $\mathrm{DE} \mathrm{kg}^{-1}$ and the same rate between calcium:phosphorus. Based on these results, $0.75 \%$ of available phosphorus in Nile tilapia fingerlings diets determines a better growth performance and body chemical composition.

Key words: mineral, phytate, fishes, nutrition, Oreochromis niloticus.

\section{INTRODUÇÃO}

A criação de peixes em sistemas intensivo e superintensivo vem aumentando de forma expressiva e, maior atenção tem sido dada ao alimento utilizado, uma vez que a ração é o único alimento disponível aos peixes. A determinação das exigências nutricionais nas diferentes fases de criação da tilápia do Nilo (Oreochromis niloticus) é essencial para o correto manejo alimentar, para minimizar custos de formulação, para diminuir o impacto ambiental e para melhorar os resultados produtivos.

Peixes na fase inicial de desenvolvimento exigem elevados níveis de nutrientes. Os alimentos de origem animal são muito utilizados nessas formulações por apresentarem maiores níveis proteína e fósforo.

${ }^{1}$ Departamento de Nutrição Animal, Faculdade de Medicina Veterinária e Zootecnia (FMVZ), Universidade Estadual Paulista (Unesp), CP 560, 18618-000, Botucatu, SP, Brasil. E-mail: epezzato@fca.unesp.br. Autor para correspondência.

${ }^{2}$ Curso de Zootecnia, FMVZ, Unesp, Botucatu, SP, Brasil.

${ }^{3}$ Departamento de Nutrição Animal, FMVZ, Unesp, Botucatu, SP, Brasil.

${ }^{4}$ Programa de Pós-graduação em Zootecnia, FMVZ, Unesp, Botucatu, SP, Brasil. 
Dentre os minerais, o fósforo é o que apresenta maior importância para o crescimento, a mineralização óssea e o metabolismo de lipídios. Embora a farinha de peixe contenha altos níveis de fósforo, sua disponibilidade para a tilápia do Nilo é de apenas 27,1\%, o que resulta em excesso de fósforo excretado para o meio ambiente (MIRANDA et al., 2000a). Alguns alimentos de origem vegetal apresentam boa digestibilidade, menor custo, são disponíveis no mercado e podem substituir a farinha de peixe da ração (FURUYA et al., 2001).

A exigência em fósforo para as diversas espécies de peixes varia entre 0,25 e 1,0\% da dieta, sendo esta variação relacionada à fase de crescimento (DOUGALL et al., 1996). Tal exigência depende das características do sistema digestório e da fonte desse mineral. A assimilação de fósforo a partir do alimento é 200 vezes maior que o absorvido da água (HEPHER, 1990) e sua suplementação é necessária, principalmente, quando se utilizam alimentos de origem vegetal, nos quais este mineral se apresenta na forma de fósforo fítico ou fitato, forma esta não disponível aos peixes (VIELMA \& LALL, 1998).

O objetivo deste estudo foi, por meio da avaliação do crescimento e da composição química da carcaça, determinar a exigência em fósforo disponível para alevinos de tilápia do Nilo recém-revertidos sexualmente.

\section{MATERIAL E MÉTODOS}

Essa pesquisa foi realizada na Unesp Universidade Estadual Paulista no Laboratório de Nutrição de Organismos Aquáticos da FMVZ, Botucatu, SP.

Trinta aquários com capacidade para seis litros foram montados em sistema de recirculação de água, com controle de temperatura e filtro biológico. $\mathrm{O}$ fluxo de água foi de $0,1 \mathrm{~L} \mathrm{~min}{ }^{-1} \mathrm{e}$ a temperatura da água controlada para permanecer constante em $26,0^{\circ} \mathrm{C}$, com fotoperíodo de 12 horas luz e de 12 horas escuro. Os parâmetros de qualidade de água se mantiveram estáveis, na faixa recomendada para as tilápias por POPMA \& GREEN (1990), com médias de $\mathrm{pH}$, temperatura, oxigênio dissolvido e amônia de $7,5 \pm 0,5^{\circ} \mathrm{C}$; $26,5 \pm 0,5^{\circ} \mathrm{C} ; 7,2 \pm 1,0 \mathrm{mg} \mathrm{L}^{-1}$ e, $0,05 \pm 0,0 \mathrm{mg} \mathrm{L}^{-1}$, respectivamente.

As diferentes rações experimentais e, no final do experimento, as amostras dos peixes de cada repetição (em duplicata) foram submetidas à análise bromatológica no Laboratório de Bromatologia da FMVZ (AOAC, 1990). As concentrações de cálcio e de fósforo foram determinadas por espectrometria de absorção atômica (FREIRE et al., 2001), no Laboratório de Química da Unesp, Botucatu. A composição químicobromatológica dos diferentes alimentos utilizados nas rações encontra-se na tabela 1 e os valores da composição percentual e químico-bromatológica das rações na tabela 2.

As rações foram formuladas segundo as recomendações para a espécie de MIRANDA et al. (2000a,b), FURUYA et al. (2001) e GONÇALVES et al. (2004), com níveis crescentes de fósforo disponível, contendo 30,0\% de proteína digestível, 3300kcal ED $\mathrm{kg}^{-1}$ de ração e mesma relação cálcio:fósforo disponível. Destas, cinco continham os diferentes níveis de fósforo disponível (0,25; 0,5; 0,75; 1,0 e 1,25\%) e uma ração (controle) estava isenta de suplementação de fósforo inorgânico (Tabela 2). Os ingredientes foram moídos e padronizados ao diâmetro de $0,5 \mathrm{~mm}$; as misturas foram homogeneizadas, submetidas à extrusão.

Os peixes (180) com peso inicial médio de 0,27 $\pm 0,04 \mathrm{~g}$ foram distribuídos em trinta aquários (seis peixes/aquário), compondo seis tratamentos e cinco repetições. Os peixes foram alimentados à vontade seis vezes ao dia, de forma a não ocorrerem sobras de ração nos aquários. Ao final do experimento, os peixes foram anestesiados em benzocaína (1,0g em 15,0L de água), pesados, sacrificados e congelados. Foram avaliados: ganho em peso, consumo de ração aparente, conversão

Tabela 1 - Composição químico-bromatológica dos alimentos utilizados nas rações experimentais (base na matéria natural).

\begin{tabular}{|c|c|c|c|c|c|c|c|}
\hline Ingrediente & MS (\%) & PB (\%) & $\mathrm{EB}\left(\mathrm{kcal} \mathrm{kg}^{-1}\right)$ & $\mathrm{EE}(\%)$ & FB (\%) & $\mathrm{P}(\%)$ & $\mathrm{Ca}(\%)$ \\
\hline Farelo de soja & 87,71 & 45,35 & 4269 & 1,09 & 5,78 & 0,54 & 1,07 \\
\hline Milho & 88,55 & 7,42 & 4616 & 1,40 & 0,68 & 0,12 & 0,20 \\
\hline Arroz (quirera) & 90,00 & 8,00 & 3907 & 1,12 & 0,31 & 0,21 & 0,07 \\
\hline Levedura & 91,00 & 49,17 & 4170 & 21,87 & 1,25 & 0,15 & 0,16 \\
\hline Celulose & 90,00 & - & - & - & 75,00 & - & - \\
\hline Óleo de soja & 99,30 & - & 9540 & 99,00 & - & - & - \\
\hline Fosfato bicálcico & - & - & - & - & - & 18,50 & 24,80 \\
\hline Calcário & - & - & - & - & - & - & 38,40 \\
\hline
\end{tabular}


Tabela 2 - Composição percentual e químico-bromatológica das rações experimentais (base na matéria natural).

\begin{tabular}{|c|c|c|c|c|c|c|}
\hline \multirow{2}{*}{ Ingrediente } & \multicolumn{6}{|c|}{ Fósforo disponível (\%) } \\
\hline & 0,0 & 0,25 & 0,50 & 0,75 & 1,00 & 1,25 \\
\hline Farelo de soja & 60,50 & 60,50 & 60,80 & 61,00 & 61,30 & 62,00 \\
\hline Milho & 17,47 & 17,08 & 15,37 & 13,77 & 12,18 & 13,53 \\
\hline Levedura & 2,00 & 2,00 & 2,00 & 2,00 & 2,00 & 2,00 \\
\hline Quirera de arroz & 9,00 & 9,00 & 9,00 & 9,00 & 9,00 & 6,00 \\
\hline Óleo de soja & 1,20 & 1,20 & 1,80 & 2,40 & 2,90 & 3,10 \\
\hline DL-Metionina & 0,50 & 0,50 & 0,50 & 0,50 & 0,50 & 0,50 \\
\hline L-Lisina & 0,93 & 0,93 & 0,93 & 0,93 & 0,93 & 0,88 \\
\hline Celulose & 1,00 & 1,00 & 1,00 & 1,00 & 1,00 & 1,00 \\
\hline Fosfato bicálcico & 0,0 & 1,30 & 3,50 & 5,80 & 8,00 & 10,30 \\
\hline Calcário & 6,70 & 5,80 & 4,40 & 2,90 & 1,50 & 0,0 \\
\hline Suplemento Vitamínico e Mineral ${ }^{(1)}$ & 0,50 & 0,50 & 0,50 & 0,50 & 0,50 & 0,50 \\
\hline $\mathrm{NaCl}$ & 0,10 & 0,10 & 0,10 & 0,10 & 0,10 & 0,10 \\
\hline BHT (antioxidante) & 0,02 & 0,02 & 0,02 & 0,02 & 0,02 & 0,02 \\
\hline Vitamina C monofosfatada & 0,08 & 0,08 & 0,08 & 0,08 & 0,08 & 0,08 \\
\hline Total & 100,0 & 100,0 & 100,0 & 100,0 & 100,0 & 100,0 \\
\hline \multicolumn{7}{|l|}{ Composição química } \\
\hline Matéria seca (\%) & 95,11 & 94,96 & 94,57 & 95,03 & 94,25 & 94,86 \\
\hline Proteína Bruta (\%) & 34,28 & 35,59 & 34,13 & 35,86 & 34,94 & 34,71 \\
\hline Matéria Mineral (\%) & 9,95 & 9,57 & 10,60 & 11,30 & 12,24 & 12,75 \\
\hline Extrato Etéreo (\%) & 3,58 & 3,97 & 3,65 & 3,52 & 4,52 & 4,61 \\
\hline Cálcio (\%) & 3,96 & 3,52 & 3,97 & 3,82 & 3,70 & 3,41 \\
\hline Fósforo total (\%) & 0,50 & 0,55 & 1,08 & 1,39 & 2,01 & 2,16 \\
\hline
\end{tabular}

${ }^{1}$ Suplemento vitamínico e mineral (Supremais), ingrediente/kg: Vitaminas: A=1.200.000 UI; D3=200.000 UI; E=12.000 mg; K3=2.400 mg; $\mathrm{B} 1=4.800 \mathrm{mg} ; \mathrm{B} 2=4.800 \mathrm{mg} ; \mathrm{B} 6=4.000 \mathrm{mg} ; \mathrm{B} 12=4.800 \mathrm{mg} ;$ ác. fólico=1.200 mg; pantotenato de $\mathrm{Ca}=12.000 \mathrm{mg} ; \mathrm{C}=48.000 \mathrm{mg} ;$ biotina=48mg; colina=65.000mg; niacina=24.000mg; minerais: $\mathrm{Fe}=10.000 \mathrm{mg}$; $\mathrm{Cu}=600 \mathrm{mg} ; \mathrm{Mn}=4.000 \mathrm{mg} ; \mathrm{Zn=6.000} \mathrm{mg;} \mathrm{I=20} \mathrm{mg;} \mathrm{Co=2}$ $\mathrm{mg}$ e $\mathrm{Se}=20 \mathrm{mg}$;

alimentar aparente, eficiência alimentar, sobrevivência e composição química da carcaça.

O delineamento experimental utilizado foi o inteiramente casualizado e os dados foram submetidos à análise de variância (ANOVA). Quando constatada diferença entre as médias, foi realizada a análise de regressão polinomial. Para as análises estatísticas, foi utilizado o programa estatístico SAS (SAS, 1995). Os valores das variáveis em porcentagem foram transformados pela expressão $\mathrm{y}=\sqrt{x}$, sendo $\mathrm{x}$ o valor da variável expresso em percentagem.

\section{RESULTADOS E DISCUSSÃO}

\section{Desempenho}

Os resultados de ganho em peso, conversão alimentar aparente, consumo aparente e sobrevivência estão apresentados na tabela 3. Apesar da diferença nos valores de desempenho produtivo, não foram constatadas deformações aparentes na carcaça dos alevinos submetidos aos diferentes níveis de fósforo na ração, o mesmo observado por ASGARD \& SHEARER (1997) em estudo com alevinos de salmão do Atlântico (Salmo salar).

A deficiência de fósforo em peixes geralmente é expressa por baixos valores de ganho de peso e pior conversão alimentar. Esses resultados foram apresentados pelos peixes alimentados com as rações contendo níveis inferiores a $0,5 \%$ de fósforo disponível (Tabela 3), confirmando aqueles observados por ANDREWS et al. (1973) e DOVE (1976) com o bagre do canal e a carpa comum, respectivamente.

Os valores médios de ganho de peso, em função dos diferentes níveis de fósforo disponível, foram diferentes $(\mathrm{P}<0,05)$ entre os tratamentos (Tabela 3). Pode-se observar efeito quadrático por meio da equação de regressão, com estimativa de ganho em peso máximo de 3,60g com suplementação de 1,0\% de fósforo disponível. Os resultados de ganho de peso demonstraram que níveis fósforo disponível inferiores a $0,75 \%$ reduziram em até $67,5 \%$ o crescimento. Resultados semelhantes foram encontrados, com essa mesma espécie, por WATANABE et al. (1980), DATO- 
Tabela 3 - Ganho em peso (GP), conversão alimentar aparente (CAA), consumo de ração aparente (CRA), eficiência alimentar (EFA) e sobrevivência (SOB) de alevinos de tilápia do Nilo alimentados com rações contendo diferentes níveis de fósforo disponível.

\begin{tabular}{|c|c|c|c|c|c|c|c|}
\hline \multirow{2}{*}{ Variável } & \multicolumn{6}{|c|}{ Fósforo disponível (\%) } & \multirow{2}{*}{$\begin{array}{c}\text { Regressão } \\
\left(\mathrm{CV \%}{ }^{(3)} ; \mathrm{R}^{2} \%{ }^{(4)}\right)\end{array}$} \\
\hline & 0,0 & 0,25 & 0,50 & 0,75 & 1,00 & 1,25 & \\
\hline $\mathrm{GP}(\mathrm{g})^{(1)}$ & 1,18 & 1,92 & 3,02 & 3,63 & 3,61 & 3,30 & $\begin{array}{l}Y=1,0388+5,2962 x-2,7364 x^{2} \\
C V=13,91 ; R^{2}=97,64\end{array}$ \\
\hline $\mathrm{CAA}^{(1)}$ & 2,18 & 1,61 & 1,29 & 1,12 & 1,17 & 1,29 & $\begin{array}{l}Y=2,1611-2,4403 x+1,4078 x^{2} \\
C V=13,00 ; R^{2}=99,49\end{array}$ \\
\hline CRA (g) ${ }^{(2)}$ & 2,61 & 3,05 & 3,85 & 4,07 & 4,16 & 4,25 & $\begin{array}{l}Y=2,8220+1,3474 x \\
C V=12,16 ; R^{2}=86,61\end{array}$ \\
\hline $\mathrm{EFA}^{(1)}$ & 0,45 & 0,63 & 0,78 & 0,89 & 0,87 & 0,82 & $\begin{array}{l}Y=0,1695+0,3046 x-0,0326 x^{2} \\
C V=12,10 ; R^{2}=99,13\end{array}$ \\
\hline SOB (\%) & 96,60 & 86,60 & 96,60 & 93,20 & 96,60 & 89,80 & $\mathrm{CV}=10,17 ; \mathrm{R}=\mathrm{NS}$ \\
\hline
\end{tabular}

${ }^{1}$ Efeito quadrático a 5\% de significância.

${ }^{2}$ Efeito Linear a 5\% de significância

${ }^{3} \mathrm{CV} \%$ = Coeficiente de variação

${ }^{4} \mathrm{R}^{2} \%$ = Coeficiente de correlação.

\section{CAJEGAS \& YAKUPITIYAGE (1996) e MIRANDA et}

al. (2000b), demonstrando que os peixes alimentados com as rações deficientes em fósforo apresentam piores respostas de desempenho.

Os valores médios de conversão alimentar mostraram diferenças $(\mathrm{p}<0,05)$ entre tratamentos (Tabela 3). Pode-se observar efeito quadrático por meio da equação de regressão, com índice de 1,1:1,0 de conversão alimentar (suplementação estimada de $0,86 \%$ de $\mathrm{P}_{\text {disp }}$ ). Esses resultados mostram-se semelhantes aos obtidos com outras espécies de peixes, com melhora significativa na conversão alimentar com o aumento de fósforo na dieta (DATOCAJEGAS \& YAKUPITIYAGE, 1996 e ROBINSON et al., 1996) e melhor que os obtidos, com essa mesma espécie, por MIRANDA et al. (2000b), quando obtiveram conversão de 1,71 em ração com $0,75 \%$ de fósforo disponível.

Foi observado (Tabela 3) aumento linear no consumo de ração em função da maior suplementação de fósforo, com melhores respostas de ganho de peso e conversão alimentar. Esses resultados expressam a eficiência alimentar (ganho em peso/consumo de ração), a qual apresentou efeito quadrático em função dos níveis crescentes de $\mathrm{P}_{\text {disp }}$ na ração.
Análise de carcaça

Os valores (Tabela 4) de composição química da carcaça dos peixes alimentados com rações contendo diferentes níveis de fósforo disponível revelaram diferença $(\mathrm{P}<0,05)$ entre as médias de todas as variáveis analisadas (PB, EE, MM e Ca). Estas, quando submetidas à análise de regressão polinomial, apresentaram comportamento quadrático em função dos níveis de $\mathrm{P}_{\text {disp }}$, exceto para o teor de fósforo, o qual apresentou efeito linear. Pode-se constatar que os teores de proteína bruta da carcaça aumentam até o nível de suplementação de 0,61\% de $\mathrm{P}_{\text {disp }}$. Esses resultados corroboram os encontrados por SATOH et al. (1996), em estudo com trutas, quando observaram que o excesso de fósforo reduziu a síntese protéica e a disponibilidade de zinco da ração. Comportamento inverso foi observado para o extrato etéreo, o qual foi reduzido com o aumento da suplementação de fósforo. Em estudo com essa mesma espécie, MURAKAMI (1967) relatou que a suplementação de fósforo incrementou o conteúdo de proteína e reduziu o nível de lipídios nos peixes, indicando que o fósforo participa no metabolismo dos lipídios.

Pode-se observar (Tabela 4) que a carcaça dos peixes arraçoados com a ração controle (não 
Tabela 4 - Composição química da carcaça de alevinos de tilápia do Nilo alimentados com rações contendo diferentes níveis de fósforo disponível.

\begin{tabular}{|c|c|c|c|c|c|c|c|}
\hline \multirow{2}{*}{ Variável (\%) ${ }^{(1)}$} & \multicolumn{6}{|c|}{ Tratamento (\% $\left.\mathrm{P}_{\text {disp }}\right)$} & \multirow{2}{*}{$\begin{array}{l}\text { Regressão } \\
\left(\mathrm{CV} \%^{(3)} ; \mathrm{R}^{2} \%^{(4)}\right)\end{array}$} \\
\hline & 0,0 & 0,25 & 0,50 & 0,75 & 1,00 & 1,25 & \\
\hline Proteína Bruta & 65,40 & 67,78 & 69,46 & 69,83 & 65,63 & 66,16 & $\begin{array}{l}Y=65,58857+11,43829 x-9,36 x^{2} \\
C V=2,27 ; R^{2}=67,82\end{array}$ \\
\hline Extrato Etéreo & 25,60 & 22,54 & 19,72 & 18,50 & 20,25 & 20,15 & $\begin{array}{l}Y=30,174+5,1447 x-0,5907 x^{2} \\
C V=; R^{2}=94,66\end{array}$ \\
\hline Matéria Mineral & 9,09 & 9,39 & 10,74 & 12,92 & 13,13 & 12,85 & $\begin{array}{l}Y=7,25920+5,74611 x-1,87071 x^{2} \\
C V=14,34 ; R^{2}=56,15\end{array}$ \\
\hline Cálcio & 2,36 & 2,33 & 2,34 & 2,44 & 2,55 & 2,63 & $\begin{array}{l}Y=2,34914-0,14343 x-0,304 x^{2} \\
C V=4,37 ; R^{2}=96,95\end{array}$ \\
\hline Fósforo & 2,17 & 2,54 & 3,16 & 3,56 & 3,69 & 4,39 & $\begin{array}{l}Y=2,29373+1,37056 x \\
C V=14,85 ; R^{2}=62,62\end{array}$ \\
\hline
\end{tabular}

${ }^{1} 5 \%$ de significância.

${ }^{2} \mathrm{CV} \%$ = Coeficiente de variação.

${ }^{3} \mathrm{R}^{2} \%$ = Coeficiente de correlação.

suplementada com fósforo) apresentou a menor concentração de cálcio e de fósforo. Esses resultados mostram-se similares aos encontrados por DATOCAJEGAS \& YAKUPITIYAGE (1996) com a tilápia do Nilo. A suplementação de $\mathrm{P}_{\text {disp }}$ promoveu aumento linear no conteúdo de fósforo da carcaça, enquanto que, para o cálcio, esse aumento foi constatado apenas após a suplementação de $0,5 \%$ de $\mathrm{P}_{\text {disp. }}$.

Resultados diferentes aos encontrados nesse estudo foram obtidos por WATANABE et al. (1980) e por ROBINSON et al. (1987), que não observaram efeito positivo na mineralização óssea da tilápia do Nilo e da tilápia azul (Oreochromis aureus), respectivamente, quando utilizaram cálcio e fósforo suplementar. Entretanto, MIRANDA et al. (2000b) relataram que, em estudo com a tilápia do Nilo, a relação $\mathrm{Ca} / \mathrm{P}_{\text {disp }}$ 1,0:1,0 implicou maiores concentrações desses minerais na carcaça, sendo que esta relação, ocorreu quando o nível de cálcio e fósforo da ração foi de 0,5\%, devendo o fósforo estar na forma disponível.

\section{CONCLUSÕES}

Pode-se concluir que o fosfato bicálcico pode ser utilizado como fonte de fósforo em rações para alevinos de tilápia do Nilo recém revertidos e que o nível de $0,75 \%$ de fósforo disponível em rações para essa espécie após a fase de reversão sexual proporciona melhores resultados em desempenho produtivo e em mineralização óssea.

\section{AGRADECIMENTOS}

À Fundação de Amparo à Pesquisa do Estado de São Paulo-FAPESP-e à Supre Mais Produtos Bioquímicos Ltda. pelo apoio científico.

\section{REFERÊNCIAS}

ANDREWS, J.W. et al. Effects of dietary calcium and phosphorus on growth, food conversion, bone ash and hematocrit levels of catfish. Journal Nutrition, Philadelphia, v.103, p.766-771, 1973.

AOAC. Official Methods of the Association of Official Analysis Chemist. 15ed. Washington, DC, 1990. 1141p.

ASGARD, T.; SHEARER, K.D. Dietary phosphorus requirement of juvenile Atlantic salmon, Salmo salar. Aquaculture Nutrition, v.3, p.17-23, 1997.

DATO-CAJEGAS C.S.; YAKUPITIYAGE. A. The need for dietary mineral supplementation for Nile tilapia, Oreochromis niloticus, cultured in a semi-intensive culture. Aquaculture, Amsterdam, v.144, p.227-237, 1996.

DOUGALL, S.D. et al. Dietary phosphorus requirement of juveniles striped bass Morone saxatilis. Journal World Aquaculture Society, v.27, p.82-91, 1996.

DOVE, G.R. et al. Effects of three diets on growth and mineral retention of channel catfish fingerlings. Transaction American Fisheries Society, v.105, p.482-4855, 1976.

FREIRE, E.S. et al. Determinação de $\mathrm{Cr}_{2} \mathrm{O}_{3}$ utilizado como marcador de rações em fezes de peixes por GFAAS após mineralização em forno de microondas. In: ENCONTRO REGIONAL DE QUÍMICA DA SOCIEDADE BRASILEIRA DE QUÍMICA, 31., 2001, Araraquara. Anais... Araraquara: Instituto de Química da Unesp, 2001. p.73.

FURUYA, W.M. et al. Coeficientes de digestibilidade e valores de aminoácidos digestíveis de alguns ingredientes pela tilápia do Nilo (Oreochromis niloticus). Revista Brasileira de Zootecnia, Viçosa, v.30, n.4, p.1143-1149, 2001.

GONÇALVES, G.S. et al. Digestibilidade aparente e suplementação de fitase em alimentos vegetais para a tilápia do Nilo. Acta Scientiarum, Maringá, v.26, n.3, p.313-321, 2004. 
HEPHER, B. Nutrition of pond fishes. New York: Cambridge University, 1990. 407p.

MIRANDA, E.C. et al. Disponibilidade aparente de fósforo em ingredientes pela tilápia do Nilo (Oreochromis niloticus). Acta Scientiarum, Maringá, v.22, n.3, p.669-675, 2000a.

MIRANDA, E.C. et al. Relação cálcio/fósforo disponível em rações para a tilápia do Nilo (Oreochromis niloticus). Revista Brasileira de Zootecnia, Viçosa, v.29, n.6, p.2162-2171, 2000b.

MURAKAMI, Y. Studies on a cranial deformity in hatchery reared young carp. Fish Pathology, v.2, n.1, p.1-10, 1967.

POPMA, T.J.; GREEN, B.W. Sex reversal of tilapia in earthen ponds. Auburn, Alabama: International Center for Aquaculture. Alabama Agricultural Experment Station, Auburn University, 1990. 93p.
ROBINSON, E.H. et al. Dietary calcium and phosphorus requirements of Oreochromis niloticus reared in calcium-free water. Aquaculture, Amsterdam, v.64, p.267-279, 1987.

SATOH, S. et al. Influence of dietary phosphorus levels on growth on mineral availability in rainbow trout. Fisheries Science, v.62, p.483-487, 1996.

STATISTICAL ANALYSIS SYSTEMS INSTITUTE - SAS. Use's guide. 8.ed. Cary, USA: SAS ${ }^{\circledR} / \mathrm{STAT}$, SAS Institute, 1999. 365p.

VIELMA, J.; LALL. S.P. Phosphorus utilization by Atlantic salmon (Salmo salar) reared in freshwater by higher dietary calcium intake. Aquaculture, Amsterdam, v.160, p.117-128, 1998.

WATANABE, T. et al. The availability to tilapia nilotica of phosphorus in white fish meal. Bulletin Japanese Society Science Fish, v.46, p.897-899, 1980. 